AperTO - Archivio Istituzionale Open Access dell'Università di Torino

\title{
Updated genetic testing of Italian patients referred with a clinical diagnosis of primary hyperoxaluria
}

\section{This is the author's manuscript}

Original Citation:

\section{Availability:}

This version is available http://hdl.handle.net/2318/1559459

since 2017-03-31T19:35:49Z

Published version:

DOI:10.1007/s40620-016-0287-4

Terms of use:

Open Access

Anyone can freely access the full text of works made available as "Open Access". Works made available under a Creative Commons license can be used according to the terms and conditions of said license. Use of all other works requires consent of the right holder (author or publisher) if not exempted from copyright protection by the applicable law. 


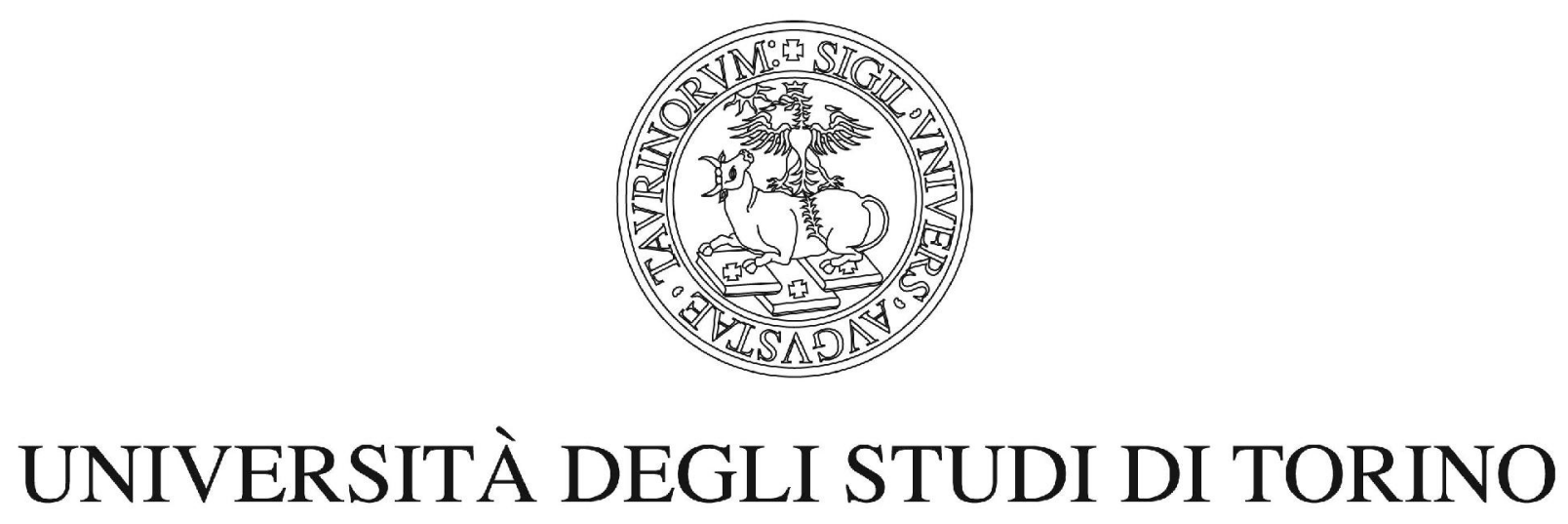

This is an author version of the contribution published on:

Questa è la versione dell'autore dell'opera:

Journal of Nephrology, First published online: 05 March 2016, 10.1007/s40620-016-

0287-4

The definitive version is available at:

La versione definitiva è disponibile alla URL:

http://link.springer.com/article/10.1007\%2Fs40620-016-0287-4 
JOURNAL OF NEPRHOLOGY

ORIGINAL ARTICLE

Updated genetic testing of Italian patients referred with a clinical diagnosis of primary hyperoxaluria.

\begin{abstract}
Alessandra Pelle ${ }^{1,2}$, Alessandra Cuccurullo ${ }^{1,2}$, Cecilia Mancini $^{3}$, Regina Sebastiano ${ }^{2}$, Giovanni Stallone $^{4}$, Susanna Negrisolo ${ }^{5}$, Elisa Benetti ${ }^{5}$, Licia Peruzzi ${ }^{6}$, Michele Petrarulo ${ }^{7}$, Mario De Marchi ${ }^{1,2}$, Martino Marangella ${ }^{8}$, Antonio Amoroso ${ }^{3}$, Daniela Giachino ${ }^{1,2}$, Giorgia Mandrile ${ }^{1}$
\end{abstract}

${ }^{1}$ University of Torino, Department of Clinical \& Biological Sciences, Orbassano, Italy

${ }^{2}$ Medical Genetics Unit, San Luigi University Hospital, Orbassano, Italy

${ }^{3}$ University of Torino, Department of Medical Sciences, Città della Salute e della Scienza, Torino, Italy

${ }^{4}$ University of Foggia, Department of Medical and Surgical Sciences, Foggia, Italy

${ }^{5}$ Pediatric Nephrology, Dialysis and Transplant Unit, Department of Pediatrics, University of Padova, Italy

${ }^{6}$ Nephrology Dialysis Transplantation Unit, Regina Margherita Children's Hospital, Città della Salute e della Scienza, Torino, Italy

${ }^{7}$ S.C. Laboratorio Centrale, A.O. Ordine Mauriziano, Torino, Italy

${ }^{8}$ Fondazione Scientifica, A.O. Ordine Mauriziano, Torino, Italy

\title{
Corresponding author:
}

Alessandra Pelle, University of Torino, Department of Clinical \& Biological Sciences, San Luigi University Hospital, Regione Gonzole 10 - 10040 Orbassano (TO) - Italy. phone: +39-0119026205, fax: +39-0116705428, genmed-dscb@unito.it, alessandraelisapelle@gmail.com 
ORIGINAL ARTICLE

\title{
Updated genetic testing of Italian patients referred with a clinical diagnosis of primary hyperoxaluria.
}

\begin{abstract}
Background: Primary hyperoxaluria (PH) is a rare autosomal recessive disease commonly arising in childhood and presenting with nephrolithiasis, nephrocalcinosis and/or chronic renal failure. Three responsible genes are known: alanine-glyoxylate aminotransferase ( $A G X T, \mathrm{PH}$ type 1), glyoxylate reductase/hydroxypyruvate reductase (GRHPR, PH type 2), and 4-hydroxy-2-oxoglutarate aldolase (HOGA1, PH type 3). In our centre at the end of 2014 molecular diagnosis of PH1 had been performed in 80 patients, while one patient received a $\mathrm{PH} 2$ diagnosis.

Material and Methods: Fifteen patients referred to our Centre and suspected to have PH on clinical grounds were negative for pathogenic variants in the entire coding sequence and exon-intron boundaries of the $A G X T$ gene. Therefore, we extended the analysis to the $A G X T$ promoter region and the GRHPR and HOGAl genes.

Results: Two patients were heterozygous for two novel $A G X T$-promoter variants (c.-647C>T, c.$424 \mathrm{C}>\mathrm{T}$ ) that were probably non pathogenic. One patient was homozygous for a novel HOGAl variant of intron 2 (c.341-81delT), whose pathogenicity predicted by in silico splicing tools was not confirmed by a minigene splicing assay in COS-7 and HEK293T cells.

Conclusion: New genetic subtypes of PH can be hypothesized in our patients, that may be caused by mutations in (an)other gene/genes encoding proteins of glyoxylate metabolism.

Alternatively, some kind of mutations (e.g. deletions/duplications, deep intronic splicing regulatory variants) could be missed in a few cases, similarly to other genetic diseases.
\end{abstract}

Keywords: $A G X T$, glyoxylate metabolism, GRHPR, $H O G A 1$, primary hyperoxaluria. 
Abbreviation footnote:

AGT: alanine-glyoxylate aminotransferase

ESRD: end stage renal disease

GFR: glomerular filtration rate

GRHPR: glyoxylate reductase/hydroxypyruvate reductase

GO: glycolate oxidase

HOGA: 4-hydroxy-2-oxoglutarate aldolase

LDH: lactate dehydrogenase

nv: normal value

$\mathrm{PH}$ : primary hyperoxaluria

PH1: primary hyperoxaluria type 1

PH2: primary hyperoxaluria type 2

PH3: primary hyperoxaluria type 3

Pox: plasma oxalate

Uox: urinary oxalate

WT: wild type 


\section{Introduction}

Primary hyperoxaluria $(\mathrm{PH})$ is a rare autosomal recessive disease (prevalence $1-3 \times 10^{-6}$ ) with onset mainly in childhood [1]. It is characterized by increased levels of urinary and plasma oxalate, nephrolithiasis and/or nephrocalcinosis leading to end stage renal disease (ESRD) and systemic oxalosis [2]. Three types of PH are currently known, but further genetic heterogeneity has been hypothesized, since in some patients the clinical suspicion of $\mathrm{PH}$ is not confirmed by molecular analysis [1].

PH1 (MIM \#259900), the most frequent and severe form, is caused by an impaired function of the alanine-glyoxylate aminotransferase enzyme (AGT), encoded by the AGXT gene (MIM *604285) [3,4]. Deficiency of glyoxylate reductase/hydroxypyruvate reductase enzyme, encoded by the GRHPR gene (MIM *604296), is responsible of PH2 (MIM \#260000) [5]. PH2 patients rarely develop systemic oxalosis and only $20 \%$ of them progress to ESRD [5-7]. A peculiar biochemical PH2 characteristic is the presence of increased urinary L-glyceric acid [8]. PH3 (MIM \#613616) is caused by mutations in the HOGAl gene (MIM*613597), that encodes the mitochondrial enzyme 4hydroxy-2-oxoglutarate aldolase (HOGA), catalysing the final step of 4-hydroxy-2-oxoglutarate catabolism to glyoxylate and pyruvate [9]. The age at onset of signs and symptoms is similar to those of PH1 and PH2, but in most PH3 patients a clinical remission is observed in the adult, despite the persistence of high oxalemia and oxaluria levels [10,11].

High 24-hour urinary oxalate (Uox) levels are suggestive of $\mathrm{PH}$ in the absence of secondary causes of hyperoxaluria, such as inflammatory bowel diseases or intestinal malabsorption [1,4]. Another important parameter is the molar urinary Oxalate/Creatinine ratio, that should always be related to the age of the subject, since higher ratios are observed in children due to lower urinary creatinine concentrations [12]. In patients with ESRD, Uox can be within the normal range or even below because of the decreased glomerular filtration; in such cases Plasma Oxalate (Pox) measurement it is preferable [1].

The assay of $A G X T$ activity on hepatic tissue, once considered as the gold standard in PH1 diagnosis, is at present rarely performed, since the less invasive genetic testing has become available. In addition, the clinical presentation of the three PH types is largely overlapping, thus genetic analysis is an important tool to differentiate the three subtypes. According to the current recommendations, a different treatment is indicated for each $\mathrm{PH}$ type: combined kidney-liver transplantation is recommended only for PH1 [3], while isolated kidney transplantation is mostly performed in $\mathrm{PH} 2$, and no specific need of transplantation has been so far reported for PH3. 
Moreover, $A G X T$ genotype can help in predicting the effectiveness of vitamin B6 supplementation [1].

Here we report the 80 Italian PH1 and the only PH2 patients currently known in our Country, collected until the end of 2014 and we describe a group of 15 patients with a clinical suspicion of PH but without a mutation in the three known genes. Moreover, we characterize the functional assay of a $H O G A l$ variant of unknown pathogenicity.

\section{Material and methods}

Major clinical criteria to suspect PH included nephrolithiasis, nephrocalcinosis and precocious age at onset of symptoms, while macro/micro hematuria and recurrent urinary infections were considered as indirect signs of renal stone disease. Glomerular filtration rate (GFR) in children was evaluated using the appropriate Schwartz's formula according to creatinine dosage assay while for adults the MDRD or CKD-EPI was employed. Urinary tract anomalies and secondary causes of hyperoxaluria, such as intestinal malabsorption and inflammatory bowel disease, were excluded. Biochemical parameters included oxalemia, oxaluria, urinary glycolate (see ref [12] for normal values) and renal function (plasma creatinine and GFR). Data about parents' consanguinity and the familiar history were collected.

\section{Sanger sequencing of AGXT, GRHPR and HOGAl genes}

Genomic DNA was extracted from peripheral blood using Maxwell System (Promega, WI, USA). Amplification and sequencing primers and protocols are available on request. Analysis of $A G X T$ was performed at first; in case of negative result GRPHR, HOGAl and the AGXT promoter-region have been analyzed.

\section{Minigene assay}

In vitro analysis of the potential splice-site HOGA1 c.341-81delT variant was performed using the pSPL3 splicing assay previously described $[10,13,14]$.

A 449 bp genomic fragment of DNA centered on the change c.341-81delT from the patient and one from a wild type (WT) control sample were amplified and the PCR products were cloned into a TAvector (pGEM-T Easy Vector - Promega, Madison, WI, USA) and transformed into DH5a bacterial cells (RBC Bioscience, Chung Ho City, Taiwan). Plasmids containing the WT or the variant 
sequence were extracted and digested with EcoRI and NotI restriction endonuclease. Inserts were sub-cloned into a pSPL3 exon-trapping vector (Life Technologies, Paisley, UK). PSPL3 minigene constructs containing the genomic change c.341-81delT and the WT sequence were extracted from DH5a bacterial cells and transfected into both COS-7 and HEK293T cells. After $24 \mathrm{~h}$, total RNA was extracted and retro-transcribed with the Cells-To-Ct kit (Life Technologies). The cDNA was amplified by standard and nested PCR and the different amplicons were separated on a TBEagarose $1.8 \%$ gel (Online Resource, protocols available upon request).

\section{Results}

Clinical and biochemical data of the 105 patients referred to our Centre were evaluated according to recent guidelines [3] and the clinical suspicion of PH was supported by clinical findings in 96 of them. A different diagnosis was established in nine patients: four were affected by secondary hyperoxaluria, three by kidney malformations; one was diagnosed as Dent and one as Pseudo-Bartter syndrome.

\section{Clinical, biochemical and genetic characteristics of $\mathrm{PH} 1$ and $\mathrm{PH} 2$ patients}

At the end of 2014 the Italian database included 80 PH1 patients (28 females and 52 males) and one PH2 patient. Thirteen patients (16\%) had consanguineous parents (information not available for 16 patients), a familiar history was reported in 30 cases $(24.6 \%)$.

Symptoms at onset were mainly nephrolithiasis (52.5\%) and nephrocalcinosis (35.2\%). Three patients were diagnosed at family screening before symptoms developed. Median age at onset was 4 years (range 0.5-11.5), median age at diagnosis was 11 years (range 4-30).

Biochemical measurements at diagnosis were not available for 11 patients. Median Pox at diagnosis was 130 umol/L (24-187; normal values (nv): <2.5 umol/L; data not available in 23 patients) and median Uox $230 \mathrm{uM} / \mathrm{mM}$ creat per day (151-435.5; data not available in 41 patients).

Liver biopsy for AGT activity measurement was available for 35 patients: mean residual AGT activity was negligible in 27 , only in $8 / 35$ (22.9\%) samples a partial AGT activity was detected (mean: $39 \pm 13 \%$ of the normal).

At the end of 2014, 55 patients had ESRD and the median age was 14 years (range 4-31). 
Ten patients had a kidney-only transplant and two of them had a second kidney-only transplant. In another six cases a second kidney transplant was combined with liver transplant. Median age at first kidney transplant was 18 years old (range 10-35). In 17 patients a combined kidney-liver transplant was performed and in one a sequential kidney-liver transplant. Median age at kidney-liver transplant was 13 years old (range 4-27). In two cases a second kidney transplant was performed after the combined kidney-liver. One patient underwent two combined kidney-liver transplants. In one case a preemptive liver transplant was performed.

As reported in literature, also in the Italian cohort the most common $A G X T$ mutation is p.Gly170Arg ( $28 \%$ of the alleles) detected in 14 patients in homozygosis, in 11 in heterozygosis with a missense mutation and in four with a null mutation.

The patient affected by PH2 suffered from recurrent nephrolithiasis since 9 years old. Neither familiar history of nephrolithiasis nor parents consanguinity was referred. Uox was $77 \mathrm{uM} / \mathrm{mM}$ creat per day at 31 years of age. Urinary L-glycerate was $4600 \mathrm{uM} / 24 \mathrm{~h}(\mathrm{nv}<30)$. His renal functional was normal until the end of 2014. Molecular analysis revealed the c.295C>T, p.Arg99* mutation in GRHPR gene in homozygosis.

Clinical, biochemical and genetic characteristics of non-PH1 non-PH2 patients

Among the fifteen mutation-negative patients (six males), eleven (73\%) had nephrolithiasis (two bilateral), five (33\%) had nephrocalcinosis and one was affected with both. The median age at onset of symptoms was 5.3 years (range: 2 months-32 years). Renal function was normal in nine; three had already progressed to ESRD; data were not available for three patients. Three patients had recurrent urinary infections, four hematuria (three of them microhematuria).

Uox was available for eight patients; in children < 12 years, mean oxaluria was $190.3 \mathrm{mmol} / \mathrm{mmol}$ creatinine (range: 106-366) while in adults $78 \mathrm{mmol} / \mathrm{mmol}$ creatinine (nv $<40 \mathrm{mmol} / \mathrm{mmol}$ creatinine [12]).

Mean Pox was available in four children $(7.3 \mu \mathrm{mol} / \mathrm{L})$ and three adults $(33.9 \mu \mathrm{mol} / \mathrm{L})$ in ESRD state. Urinary calcium in patients with preserved renal function was in the normal range.

Four patients had family history of nephrolithiasis (Online Resource table 1).

Among these patients no pathogenic mutations in the three PH genes were found.

Sequencing of the $A G X T$ promoter revealed two novel variants: one patient was heterozygous for the c. $-647 \mathrm{C}>\mathrm{T}$ variant and another patient resulted heterozygous for the c. $-424 \mathrm{C}>\mathrm{T}$ variant. 
Neither variants were reported in dbSNP141, 1000Genomes (http://www.1000genomes.org, 29/07/2015), EVS (Exome variant server, 29/07/2015), EXAC (29/07/2015) and, because they do not lie in any known regulatory site, were classified as probably non pathogenic.

A third patient carried a homozygous variant in the intron 2 of HOGA1 (c.341-81delT). This variant is not reported in EXAC Browser or EVS, but it is reported in dbSNP and 1000Genomes (http://www.1000genomes.org 29/07/2015) as a variant of unknown pathogenicity (rs570638580, MAF/minor allele: 0,0030/15).

In silico tools for splicing prediction [15-19] reported c.341-81delT as pathogenic, because it generates a novel acceptor splicing site (AG in c.341-79 position). Four out of five tools (SSF, MaxEntScan, Gene Splicer, HSF) predicted a possible damaging role of the variant, with an alternative transcript 79bp longer r.(340_341ins341-79_341-1). This transcript would cause a reading frameshift and a truncated protein product (premature stop codon in exon 3: p.(Thr115Glufs*43)).

\section{Clinical characteristics of the patient homozygous for the c.341-81delT HOGA1 variant}

The patient homozygous for the HOGAl variant suffered from nephrocalcinosis since two months of life and died at four months of respiratory failure. Pox levels were normal $(<1 \mathrm{mmol} / \mathrm{L})$, while values of oxaluria/urinary creatinine were at the upper limit of the normal range (366 $\mu \mathrm{mol} / \mathrm{mmol}$, nv for 0-6 months $325-360 \mu \mathrm{mol} / \mathrm{mmol}$ creatinine). Urinary calcium level was at the upper limit $(\mathrm{uCa} / \mathrm{uCr} 0,56 \mathrm{mmol} / \mathrm{mmol}, \mathrm{nv}$ for children $<1$ years old: $<0.4)$. The renal function at four month of life was decreased (GFR $53 \mathrm{ml} / \mathrm{min} / 1,73 \mathrm{~m}^{2}$ ). His parents were not consanguineous.

\section{HOGA1 minigene assay}

In order to substantiate the predicted effect of the HOGA1 c.341-81delT variant on splicing we set up a minigene assay by cloning the intron 2 and exon $3 \mathrm{WT}$ and variant sequences into a pSPL3 vector (Fig.1). We expected a $391 \mathrm{bp}$ product resulting from the canonical splicing of HOGAl exon 3, a 471bp product from the alternative splicing generated by the deletion of $\mathrm{T}$ in c.341-81, and a $263 \mathrm{bp}$ product resulting from the complete skipping of the inserted exon. Analysis of the cDNA showed only the presence of the two $263 \mathrm{bp}$ and $391 \mathrm{bp}$ bands, without any difference from WT (Fig. 2). 


\section{Discussion}

The genetic basis of $\mathrm{PH}$ has been widened in recent years thanks to second line studies of $A G X T$ negative patients, leading to identify $\mathrm{PH} 2$ and $\mathrm{PH} 3[1,9]$.

Nevertheless, a grey zone of cases in which suspicion based on clinical and biochemical findings is not confirmed by genetic testing still exists. The exact prevalence of this subset is currently unknown: several Authors reported the existence of undefined PH, but did not specify its prevalence or its clinical and genetic characteristics $[1,3,20]$.

In our cohort of patients, 80 resulted homozygous or compound heterozygous for $A G X T$ mutations $(83.3 \%)$ and one homozygous for a recurrent GRHPR mutation (1.1\%). The $15.6 \%$ of the subjects with clinical PH suspicion resulted negative for any $\mathrm{PH}$ mutation. A recent UK study performed in 200 patients detected mutations in AGXT, GRHPR and HOGAI in 50\%, 8\% and 8\% of the patients respectively, while in $25 \%$ of the subjects no mutations were found [21]. In the USA, the analysis of $301 \mathrm{PH}$ families revealed $\mathrm{PH} 1$ in $68.4 \%$ of them, $\mathrm{PH} 2$ in $9.3 \%$ and $\mathrm{PH} 3$ in $11.0 \%$ [20]. Compared to these results, PH1 in Italy seems to be far more frequent than the other PH types and, surprisingly, no Italian PH3 cases were identified up to now.

In two patients two novel heterozygous $A G X T$-promoter variants, c. $-647 \mathrm{C}>\mathrm{T}$ and c. $-424 \mathrm{C}>\mathrm{T}$ were identified. We considered these variants not to be pathogenic since they do not lie in any known regulatory site. The validity of this conclusion is limited by the scanty literature on the $A G X T$ promoter [22] that precludes comparison with previous studies.

In one patient we detected a homozygous HOGAl variant in intron 2 (c.341-81delT); we could not perform genetic analysis of the HOGAl variant in the patient's parents, since their DNA samples were not accessible.

The c.341-81delT variant was predicted to affect splicing by in silico analysis and to introduce a frameshift change (p.Thr115Glufs*43) in isoform 1, disruptive of HOGA enzymatic activity. Indeed, the two minor HOGA isoforms described [23] include exons 1, 5, 6, 7 (isoform 2), and exons 1, 6,7 (isoform 3) but not exons 2 and 3, and are probably not functional.

Even if the clinical characteristics of the c.341-81delT homozygous child were suggestive for PH3, a definite diagnosis cannot be established on a clinical basis alone. High urinary or plasma levels of dihydroxyglutarate and hydroxyoxoglutarate are indicative for $\mathrm{PH} 3$ [24], however the measurement of these metabolites is not yet performed in Italy.

Neither RNA nor HOGA enzymological studies were possible because further biological samples of the patient were not available. To evaluate the effect of the variant, we set up a minigene 
assay that in contrast to in silico predictions did not reveal any difference between the product of WT and c.341-81delT variant constructs. A possible explanation is that the power of the splicing site may be weaker than the canonical splicing site, so it could not be revealed by the simple minigene protocol in COS-7 and HEK293T cells.

In the Primary Hyperoxaluria Database [25] it has been reported a HOGA1 c.341-82delA variant of undetermined significance: this description and our finding of the delT at c.341-81 highlight the potential pathogenic effect of variations in this region, as pointed out also by in silico tools predictions. No functional studies are available for the c.341-82delA and we believe that this report could be a useful base for further functional studies, despite the limitations of the minigene approach.

Negative findings in our cohort of patients with a clinical diagnosis of hyperoxaluria may be ascribed to the presence of gross rearrangements (e.g. deletions/duplications), not identifiable by Sanger sequencing. However, such mutations implicate homozygosis of the SNPs haplotype and this is in contrast with our finding of heterozygous patients for several known AGXT, GRHPR and HOGA1 SNPs (Online Resource table 2).

In our patients the presence of rare deep intronic splicing mutations in the three PH genes could not be excluded, as we did not sequence the whole intronic regions. However, such mutations are rarely searched in genetic testing and their prevalence is unknown; in literature few data regarding deep intronic mutations are reported: $A G X T$ and GRHPR promoter analysis did not identify any causative mutations, while no Author has analyzed the whole intronic regions of the three PH genes $[22,26]$.

In four out of 15 cases we carried out genetic analysis on the basis of suggestive clinical history alone, although neither plasma nor urinary oxalate determinations were available since the collection was difficult; in these patients, secondary causes were excluded and a genetic etiology could be hypothesized because of their early onset of nephrolithiasis/nephrocalcinosis.

These large number of undefined patients without a definitive diagnosis (11 patients) could be explained by other nephropathies or by the hypothesis of (an)other PH type(s), as postulated by several Authors $[10,1,20]$, whose responsible gene/s is/are still unknown.

Conversely, a putatively causative homozygous or compound heterozygous genotype may not always lead to phenotypic expression, and a recent extensive population genetic screen suggested an unexpectedly high frequency of such asymptomatic/underdiagnosed cases [20].

Oxalate metabolism is complex and not fully described, it is possible that more causative or modifier genes exist [1]. As candidates, the three human lactate dehydrogenases ( $L D H A, L D H B$, 
$L D H C$ ) have been proposed, because of their important role in excessive oxalate production: $\mathrm{LDH}$ is able to convert glyoxylate to oxalate, glyoxylate to glycolate and hydroxypyruvate to L-glycerate, using $\mathrm{NAD}(\mathrm{H})$ as cofactor [27]. Genetic variants in these three genes could influence the total LDH activity and the oxalate accumulation in $\mathrm{PH}$ patients.

Another candidate involved in the pathogenesis of $\mathrm{PH}$ is the hepatocytes peroxisomal enzyme Glycolate Oxidase, (GO, encoded by HAO1 gene, OMIM *605023), also known as "hydroxyacid oxidase 1". This enzyme catalyses the oxidation of glycolate to glyoxylate, the AGT substrate. The absence of GO activity causes isolated glycolic aciduria [28], but it could be hypothesized that hypermorphic genetic $H A O l$ variants could modulate the enzyme activity leading to a different production of glyoxylate, responsible of the variable phenotype in $\mathrm{PH}$ patients.

Finally, even a multigenic PH inheritance has been suggested [10,29], on the basis of the wide clinical variability among patients with the same AGXT genotype [30]. Therefore, further studies are needed to evaluate the relative contribution of genotype and environment in the development of the clinical phenotype.

To explore this hypothesis and discover novel genes involved in oxalate metabolism, whole exome sequencing and SNPs array techniques and also further phenotype-genotype studies are needed.

In conclusion, this study is the first report of HOGAl genetic analysis in an Italian population. Despite PH3 being frequently reported both in Europe and in USA [10,20,21], no PH3 Italian cases were so far identified. It could be possible that some PH3 cases are missed because of the clinical remission of $\mathrm{PH} 3$ in adult age.

In this study the analysis of GRHPR and HOGAl genes after AGXT analysis did not increase the sensitivity of PH genetic testing. This negative report should prompt a closer interaction between clinicians and geneticians in evaluating the clinical and biochemical data and we propose a multiple step diagnostic algorithm (Fig 3) based on clinical and biochemical data to achieve this goal. 


\section{Compliance with ethical standard}

\section{Ethical approval:}

All procedures performed in studies involving human participants were in accordance with the ethical standards of the institutional and/or national research committee and with the 1964 Helsinki declaration and its later amendments or comparable ethical standards.

This article does not contain any studies with animals performed by any of the authors.

Informed consent:

Informed consent was obtained from all individual participants included in the study. 


\section{Figures and legend}

Fig.1 Minigene assay: the WT or mutant fragments of the human HOGAl gene containing exon 3 were cloned into the pSPL3 splicing vector to construct plasmids pSPL3-HOGA1-Exon3-WT and pSPL3-HOGA1-delT-Exon3. White boxes mark the pSPL3 exons A and B (derived from the Bglobin gene) and HOGA1 exon 3, and the adjacent black lines depict the flanking intronic sequence of pSPL3. The grey box represents the 79bp insertion expected from the generation of a novel splice-site by the c.341-81delT variant. The interrupted black lines mark the intronic sequences expected to be spliced out

Fig. 2 1,8\% agarose gel electrophoresis of the WT and c.341delT (delT) PCR products

Fig. 3 Proposed algorithm for the diagnosis of primary hyperoxaluria. PH: Primary hyperoxaluria, Uox: Urinary Oxalate; Pox: Plasma Oxalate 


\section{References:}

1. Beck BB, Hoyer-Kuhn H., Goebel H., et al. (2013) Hyperoxaluria and systemic oxalosis: an update on current therapy and future directions Expert Opin Investig Drugs 22 (1):117-129

2. Leumann E, Hoppe B. (2001) The primary hyperoxalurias. J Am Soc Nephrol 12 (9):1986-1993

3. Cochat P, Hulton S.A., Acquaviva C., et al. (2012) Primary hyperoxaluria Type 1: indications for screening and guidance for diagnosis and treatment. Nephrol Dial Transplant 27 (5):1729-1736

4. Hoppe B, Beck B.B., Milliner D.S. (2009) The primary hyperoxalurias. Kidney Int 75 (12):12641271

5. Cregeen DP, Williams E.L., Hulton S, Rumsby G (2003) Molecular Analysis of the Glyoxylate Reductase (GRHPR) Gene and Description of Mutations Underlying Primary Hyperoxaluria Type 2 Hum Mutat 22 (6):497

6. Milliner DS, Wilson, D.M., Smith, L.H. (2001) Phenotypic expression of primary hyperoxaluria: comparative features of types I and II. Kidney Int 59 (1):31-36

7. Marangella M, Petrarulo M, Cosseddu D (1994) End-stage renal failure in primary hyperoxaluria type 2. N Engl J Med 330 (23):1690

8. Williams H, Smith L. (1968) L-glyceric aciduria: a new genetic variant of primary hyperoxaluria. . N Enl J Med 278:233-239

9. Belostotsky R, Seboun E., Idelson G.H., et al. ( 2010) Mutations in DHDPSL are responsible for primary hyperoxaluria type III. Am J Hum Gen 87 (3):392-399

10. Beck B, Baasner A., Buerscher A., et al (2012) Novel findings in patients with primary hyperoxaluria type III and implications for advanced molecular testing strategies. Eur J Hum Genet 21 (2):162-172

11. Allard L, Cochat, P., Leclere, A.L., et al (2015) Renal function can be impared in children with primary hyperoxaluria type 3. Pediatr Nephrol 30 (10):1807-1813

12. Milliner DS (2005) The Primary Hyperoxalurias: An Algorithm for Diagnosis. Am J Nephrol 25 (2):154-160

13. Cavalieri S, Pozzi, E., Gatti, A.R., Brusco, A. (2012) Deep intronic ATM mutation detected by genomic resequencing and corrected in vitro by antisense morpholino oligonucleotide (AMO). Eur J Hum Genet 21 (7):774-778

14. Mancini C, Vaula G., Scalzitti L., et al. (2012) Megalencephalic leukoencephalopathy with subcortical cysts type 1 (MLC1) due to a homozygous deep intronic splicing mutation (c.895$226 \mathrm{~T}>\mathrm{G}$ ) abrogated in vitro using an antisense morpholino oligonucleotide. Neurogenetics 13 (3):205-214

15. SpliceSiteFinder (SSF) http://www.genet.sickkids.on.ca/ ali/splicesitefinder.html.

16. GeneSplicer http://www.cbcb.umd.edu/software/GeneSplicer/gene_spl.shtml.

17. NNSplice http://www.fruitfly.org/seq_tools/splice.html.

18. HumanSplicingFinder (HSF) http://www.umd.be/HSF/.

19. MaxEntScan http://genes.mit.edu/burgelab/maxent/Xmaxentscan_scoreseq.html.

20. Hopp K, Cogal A.G., Bergstrahl, E.J., et al (2015) Phenotype-genotype correlations and estimated carrier frequencies of primary hyperoxaluria. J Am Soc Nephrol 26 (10):2559-2570

21. Williams EL, Bagg, E.A., Mueller, M. et al (2015) Performance evaluation of Sanger sequencing for the diagnosis of primary hyperoxaluria and comparison with targeted next generation sequencing. Mol Genet Genomic Med 3 (1):69-78

22. Sato M, Toné S., Ishikawa T., et al (2002) Functional analysis of the 5'-Flanking region of the human alanine:glyoxylate aminotransferase gene AGXT. Biochimica et Biophysica Acta 1574 (2):205-209

23. Bunker RD, Loomes K.M., Baker E.N, (2012) Purification, crystallization and preliminary crystallographic analysis of human dihydrodipicolinate synthase-like protein (DHDPSL) Acta Cryst $68(\mathrm{Pt}$ 1):59-62 
24. Clifford-Mobley O, Hewitt, L., Rumsby, G. (2015) Simultaneous analysis of urinary metabolites for preliminary identification of primary hyperoxaluria. Ann Clin Biochem:pii: 0004563215606158

25. PrimaryHyperoxaluriaMutationDatabase www.uclh.nhs.uk/OURSERVICES/SERVICEAZ/PATHBIOMED/CBIO/Pages/Phmdatabase.aspx.

26. Fu Y, Rope R., Fargue S., et al (2014) A mutation creating an out-of-frame alternative translation initiation site in the GRHPR 5'UTR causing primary hyperoxaluria type II. Clin Genet 88 (5):494-498

27. Mdluli K, Booth, M. P., Brady, R. L., Rumsby, G. (2005) A preliminary account of the properties of recombinant human glyoxylate reductase (GRHPR), LDHA and LDHB with glyoxylate, and their potential roles in its metabolism. Biochim Biophys Acta 1753 (2):209-216

28. Frishberg Y, Zeharia, A., Lyakhovetsky, R., et al (2014) Mutations in HAO1 encoding glycolate oxidase cause isolated glycolic aciduria. J Med Genet 51 (8):526-529

29. Monico CG, Rossetti S., Belostotsky R., et al. (2011) Primary hyperoxaluria type III gene HOGA1 (formerly DHDPSL) as a possible risk factor for idiopathic calcium oxalate urolithiasis. Clin J Am Soc Nephrol 6 (9):2289-2295

30. Mandrile G, van Woerden CS, Berchialla P et al (2014) Data from a large European study indicate that the outcome of primary hyperoxaluria type 1 correlates with the AGXT mutation type. Kidney Int 86 (6):1197-1204 

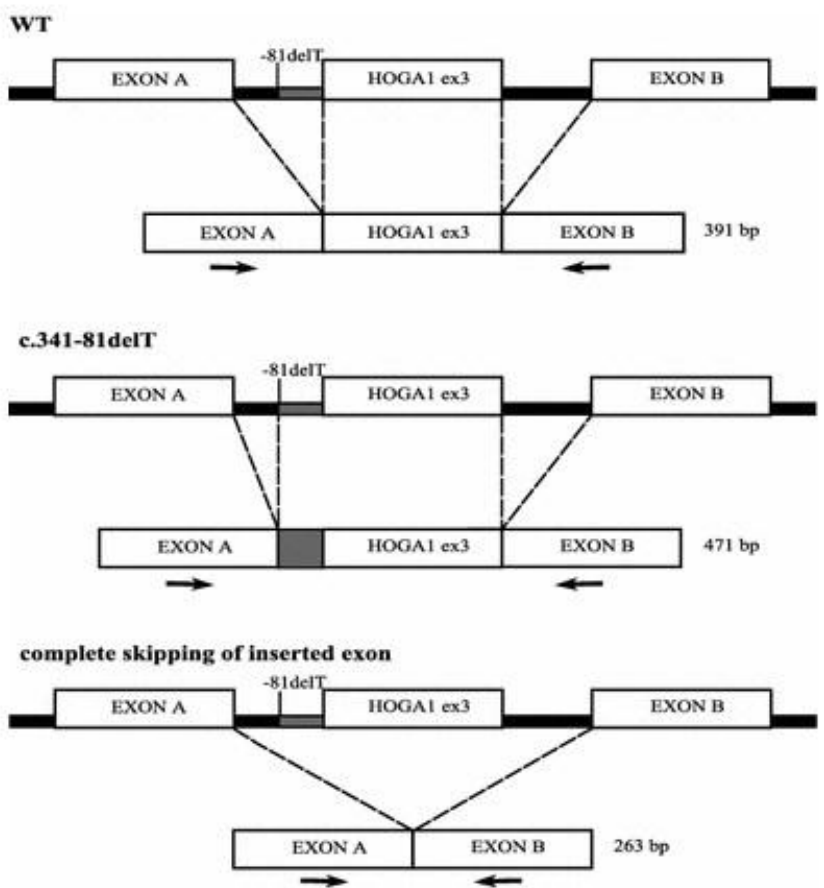

Fig. 1

Minigene assay: the WT or mutant fragments of the human HOGA1 gene containing exon 3 were cloned into the pSPL3 splicing vector to construct plasmids pSPL3-HOGA1-Exon3-WT and pSPL3-HOGA1-delT-Exon3. White boxes mark the pSPL3 exons A and B (derived from the B-globin gene) and HOGA1 exon 3 , and the adjacent black lines depict the flanking intronic sequence of pSPL3. The grey box represents the $79 \mathrm{bp}$ insertion expected from the generation of a novel splice-site by the c.341-81delT variant. The interrupted black lines mark the intronic sequences expected to be spliced out 


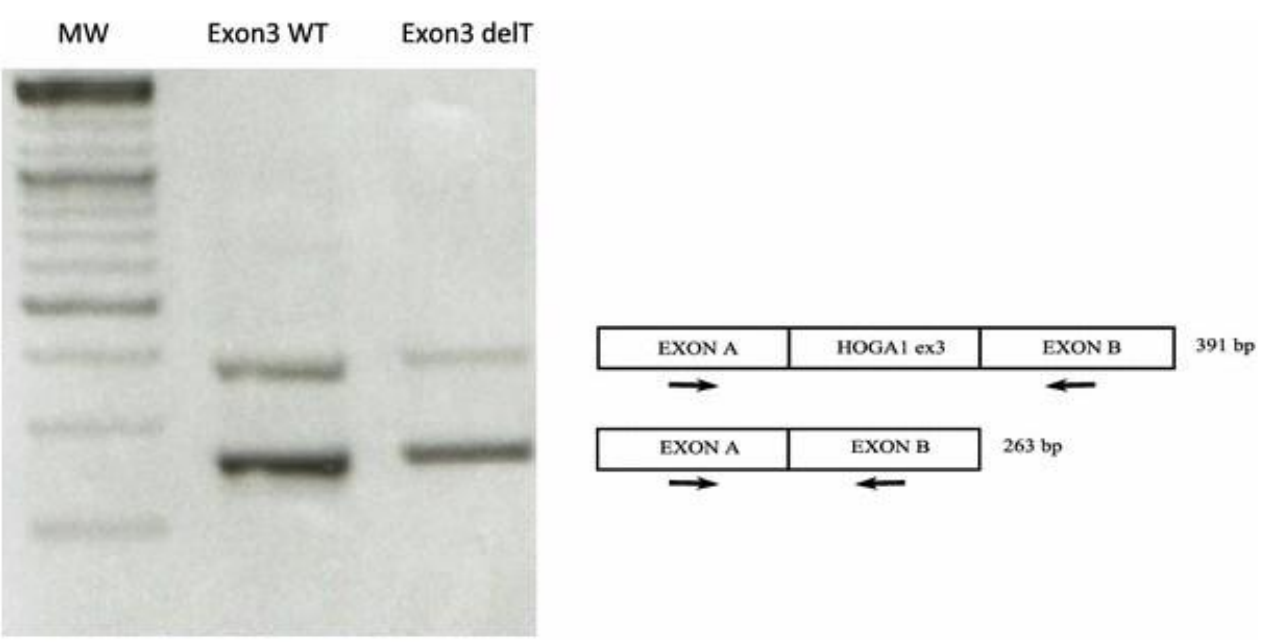

Fig. 2

$1.8 \%$ agarose gel electrophoresis of the WT and c. 341delT (delT) PCR products 


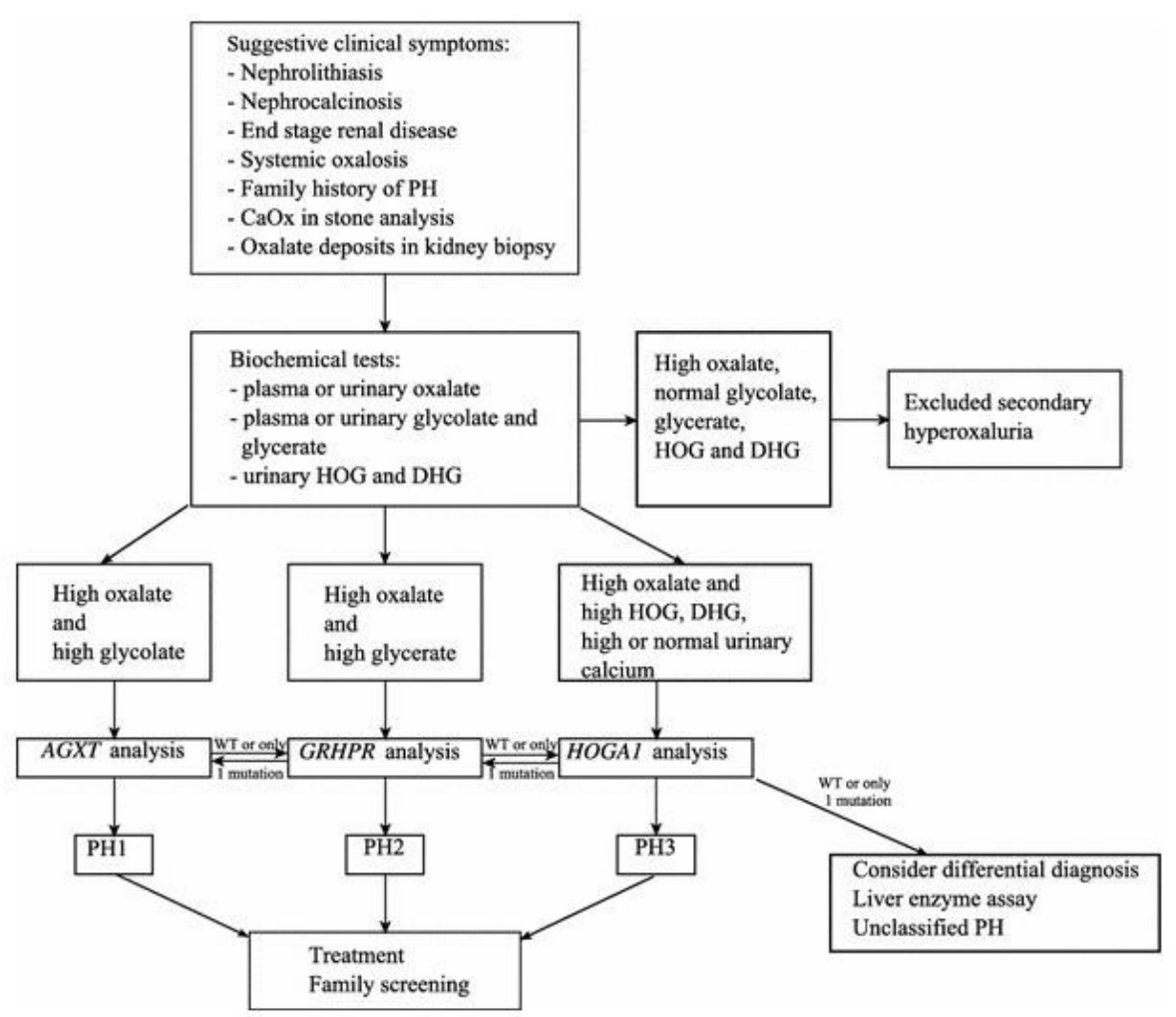

Fig. 3

Proposed algorithm for the diagnosis of primary hyperoxaluria. PH Primary hyperoxaluria, Uox urinary oxalate, Pox plasma oxalate 


\section{SUPPLEMENTARY}

Minigene assay

A 449 bp genomic fragment of DNA centered on the genomic change c.341-81delT and from a WT control sample was amplified, using the following primers:

Forward 5'-ACTGGAATTCGTGGGTGGATGTGCAGGAT-3'

Reverse 5'-GGTCAGGTAAGCCTCCCAAT-3'.

\section{Nested PCR}

A nested PCR of the c-DNA WT and c-DNA variant products was performed using the following primers: Fw 5'-AGTCGACCCAGCACTGAG; Rev 3'-CCAGGTCTTGGTGTAGTGG.

Amplicons were separated on a TBE-agarose $1,8 \%$ gel and visualized after GelRed staining, the sequences were checked. 
Table 1 Clinical characteristics of patients. Y: years, mo: months, nd: not detected

\begin{tabular}{|c|c|c|c|c|c|c|c|c|}
\hline $\begin{array}{c}\text { ID } \\
\text { patient }\end{array}$ & Sex & Age & $\begin{array}{c}\text { Age at } \\
\text { onset }\end{array}$ & ESRD & \begin{tabular}{|c|} 
Uox \\
uMol/mMol \\
creatinine
\end{tabular} & $\begin{array}{c}\text { Age at } \\
\text { Uox } \\
\text { dosage }\end{array}$ & $\begin{array}{c}\text { Pox } \\
\text { uMol/L }\end{array}$ & Clinical characteristics \\
\hline 1 & $\mathbf{M}$ & $44 \mathrm{y}$ & $32 \mathrm{y}$ & yes & nd & - & 80 & $\begin{array}{l}\text { bilateral nephrolithiasis, vascular } \\
\text { calcifications, familiarity for nephrolithiasis }\end{array}$ \\
\hline 2 & $\mathbf{F}$ & $13 \mathrm{y}$ & $9 \mathrm{y}$ & no & 70 & $9 \mathrm{y}$ & 5,6 & nephrolithiasis \\
\hline 3 & $\mathbf{M}$ & $46 \mathrm{y}$ & $3 \mathrm{y}$ & yes & nd & - & 16 & nephrolithiasis since 3 years old, mono kidney \\
\hline 4 & $\mathbf{M}$ & $4 \mathrm{y}$ & nd & nd & nd & - & nd & nephrocalcinosis \\
\hline 5 & $\mathbf{F}$ & $6 y$ & $6 \mathrm{mo}$ & no & 106 & $2 \mathrm{y}$ & 4 & $\begin{array}{l}\text { nephrolithiasis, hematuria } \\
\text { recurrent urinary infections }\end{array}$ \\
\hline 6 & $\mathbf{M}$ & $\begin{array}{c}\text { deceased } \\
\text { at } 4 \mathrm{mo}\end{array}$ & $2 \mathrm{mo}$ & no & 366 & $2 \mathrm{mo}$ & nd & nephrocalcinosis \\
\hline 7 & $\mathbf{F}$ & $16 \mathrm{y}$ & $9 \mathrm{y}$ & no & 86 & $13 \mathrm{y}$ & nd & nephrolithiasis, pyelonephritis \\
\hline 8 & $\mathbf{F}$ & $3 y$ & $4 \mathrm{mo}$ & no & 115 & $8 \mathrm{mo}$ & 10 & $\begin{array}{l}\text { nephrolithiasis, nephrocalcinosis, } \\
\text { microhematuria, crystalluria }\end{array}$ \\
\hline 9 & $\mathbf{F}$ & $5 \mathrm{y}$ & $2 y$ & no & 180 & $3 y$ & nd & $\begin{array}{l}\text { nephrolithiasis } \\
\text { familiarity for nephrolithiasis }\end{array}$ \\
\hline 10 & M & $2 \mathrm{y}$ & $6 \mathrm{mo}$ & no & 205 & $1 \mathrm{y}$ & nd & $\begin{array}{l}\text { bilateral nephrolithiasis, hematuria, familiarity } \\
\text { for nephrolithiasis }\end{array}$ \\
\hline 11 & $\mathbf{M}$ & $37 \mathrm{y}$ & $7 \mathrm{y}$ & yes & nd & - & nd & nephrolithiasis \\
\hline 12 & $\mathbf{F}$ & $3 y$ & $1 \mathrm{y}$ & no & 190 & $2 y$ & 6 & nephrolithiasis, hematuria \\
\hline 13 & $\mathbf{M}$ & $2 \mathrm{y}$ & $14 \mathrm{mo}$ & no & 170 & 14 mo & 9 & $\begin{array}{l}\text { nephrocalcinosis, familiarity for } \\
\text { nephrolithiasis }\end{array}$ \\
\hline 14 & $\mathbf{F}$ & $13 \mathrm{y}$ & $9 y$ & no & nd & - & nd & $\begin{array}{l}\text { nephrolithiasis, left nephrectomy for } \\
\text { pyonephrosis }\end{array}$ \\
\hline 15 & $\mathbf{M}$ & $1 \mathrm{y}$ & $2 \mathrm{mo}$ & nd & nd & - & nd & $\begin{array}{l}\text { nephrocalcinosis at } 3 \mathrm{mo} \text {, recurrent urinary } \\
\text { infections since } 2 \text { mo }\end{array}$ \\
\hline
\end{tabular}


Table 2 Results of genetic analysis for each patient; common SNPs founded in heterozygosis are reported in order to demonstrate the presence of both alleles for the heterozygous exons. AGXT haplotype: M major, m minor. Ex exon. WT: wild type. SNP: single nucleotide polymorphism. UV: unknown [pathogenicity] variant. SNPs code reference in dbSNP (http://www.ncbi.nlm.nih.gov/SNP/).

\begin{tabular}{|c|c|c|c|c|c|c|c|c|}
\hline \multirow{3}{*}{$\begin{array}{c}\text { Patient } \\
\text { ID } \\
1\end{array}$} & \multicolumn{2}{|c|}{$A G X T$} & \multicolumn{3}{|c|}{ GRHPR } & \multicolumn{3}{|c|}{ HOGA1 } \\
\hline & Haplotype & $\begin{array}{c}\text { Exons in } \\
\text { homozygosis }\end{array}$ & Result & SNP in heterozygosis & $\begin{array}{c}\text { Exons in } \\
\text { homozygosis }\end{array}$ & Result & $\begin{array}{c}\text { SNP in } \\
\text { heterozygosis }\end{array}$ & $\begin{array}{c}\text { Exons in } \\
\text { homozygosis }\end{array}$ \\
\hline & $\mathrm{Mm}$ & $\operatorname{ex~} 3,4,5$ & WT & rs10973332CA & ex 2-9 & WT & $\begin{array}{l}\text { rs11817730AG } \\
\text { rs7078003CT } \\
\text { rs2297644CT }\end{array}$ & ex $1,3,5,7$ \\
\hline 2 & MM & all & WT & - & all & WT & - & all \\
\hline 3 & $\mathrm{Mm}$ & ex 3-5; 7-9 & WT & $\begin{array}{l}\text { rs35891798delG } \\
\text { rs72275009insCT }\end{array}$ & ex 2-8 & WT & - & all \\
\hline 4 & $\mathrm{Mm}$ & ex 3-5; 7-9 & WT & $\begin{array}{l}\text { rs10973332CA } \\
\text { rs72275009insCT }\end{array}$ & ex 2-8 & WT & rs11527932GT & ex $1,2,3,4,5,7$ \\
\hline 5 & $\mathrm{MM}$ & ex $1,2,6,10$ & WT & rs72275009insCT & ex 2-8 & WT & - & all \\
\hline 6 & $\mathrm{MM}$ & ex $1-5,7-10$ & WT & rs72275009insCT & ex 1-8 & $\mathbf{U V}$ & - & all \\
\hline 7 & MM & ex $1-5,7-10$ & WT & - & all & WT & $\begin{array}{l}\text { rs11817730AG } \\
\text { rs7078003CT } \\
\text { rs 297644CT } \\
\text { rs12261752AC }\end{array}$ & ex $1,3,5,6$ \\
\hline 8 & MM & ex $1-5,7-10$ & WT & - & all & WT & $\begin{array}{l}\text { rs75929214CT } \\
\text { rs12261752CA }\end{array}$ & ex $1,2,3,5,6$ \\
\hline 9 & MM & $\operatorname{ex~} 2,3,5-10$ & WT & $\begin{array}{l}\text { rs35891798etdelG } \\
\text { rs76299266GA }\end{array}$ & ex 2-8 & WT & rs12261752CA & ex $1,2,3,5,6$ \\
\hline 10 & $\mathrm{Mm}$ & ex $3,5,7,8,9$ & WT & rs72275009insCT & ex $1-8$ & WT & $\begin{array}{l}\text { rs75929214CT } \\
\text { rs2297643AG } \\
\text { rs12261752CA }\end{array}$ & ex $1,2,3,6$ \\
\hline
\end{tabular}




\begin{tabular}{|c|c|c|c|c|c|c|c|c|}
\hline \multirow{2}{*}{$\begin{array}{l}\text { Patient } \\
\text { ID }\end{array}$} & \multicolumn{2}{|c|}{$A G X T$} & \multicolumn{3}{|c|}{ GRHPR } & \multicolumn{3}{|c|}{ HOGAI } \\
\hline & Haplotype & $\begin{array}{c}\text { Exons in } \\
\text { homozygosis }\end{array}$ & Result & SNP in heterozygosis & $\begin{array}{c}\text { Exons in } \\
\text { homozygosis }\end{array}$ & Result & $\begin{array}{c}\text { SNP in } \\
\text { heterozygosis }\end{array}$ & $\begin{array}{c}\text { Exons in } \\
\text { homozygosis }\end{array}$ \\
\hline 11 & $\mathrm{MM}$ & all & WT & - & all & WT & $\begin{array}{l}\text { rs2297643AG } \\
\text { rs115279832GT } \\
\text { rs } 12261752 \mathrm{CA}\end{array}$ & ex $1,2,3,4$ \\
\hline 12 & $\mathrm{MM}$ & ex 2-3; 6-10 & WT & $\begin{array}{l}\text { rs35891798delG } \\
\text { rs72275009insCT }\end{array}$ & ex 2-8 & WT & - & all \\
\hline 13 & $\mathrm{MM}$ & ex $1,6,11$ & WT & - & all & WT & rs75929214CT & ex $1,2,3,5,6,7$ \\
\hline 14 & $\mathrm{MM}$ & ex $1-4 ; 7-10$ & WT & - & all & WT & $\begin{array}{l}\text { rs75929214CT } \\
\text { rs74155508GC }\end{array}$ & ex $1,2,5,6,7$ \\
\hline 15 & MM & ex $1-5 ; 7-10$ & WT & - & all & WT & $\begin{array}{l}\text { rs11817730AG } \\
\text { rs75929214CT } \\
\text { rs7078003CT } \\
\text { rs } 2297644 C T\end{array}$ & ex $1,3,5,6,7$ \\
\hline
\end{tabular}

